\title{
The Mental Health Condition of Manufacturing Front-line Workers: The Interrelationship of Personal Resources, Professional Tasks and Mental Health
}

\author{
Qian Zhang ${ }^{1}$, Zhanwu Zhang ${ }^{2}$ and Mingbo Sun ${ }^{1, *}$ \\ ${ }^{1}$ School of Management Engineering, Zhengzhou University, Zhengzhou, China \\ ${ }^{2}$ Hong Fu Jin Precision Electronics (Zhengzhou) Co. Ltd, Zhengzhou, China \\ Corresponding Email: sunmingbo@zzu.edu.cn
}

\begin{abstract}
Manufacturing front-line workers were more likely to experience mental health problems. Personal resources and professional tasks were the major factors of workers' mental health. Therefore, this study was to explore the interrelationship of these three key factors. A questionnaire including the revised Occupational Stress Inventory (OSI-R) and the Symptom Checklist (SCL-90) covered 480 manufacturing front-line workers to measure their personal resources, professional tasks and mental health. Results showed that among manufacturing front-line workers, the status of mental health and professional tasks were below the national average level, and the personal resources were relatively deficient as well. Correlation analysis indicated a negative relation between the indicators of mental health and professional tasks (except responsibility), while personal resources and mental health were significantly positive correlation. These findings suggested that personal resources and professional tasks were highly related to mental health in manufacturing front-line workers.
\end{abstract}

Keywords. Mental health, Personal resources, Professional tasks, Front-line workers.

\section{Introduction}

With the economic globalization, China has become the "global factory". The total number of manufacturing workers has increased from 32.1 million in 2005 to 52.4 million in 2014 [1]. At present, most companies only focus on material welfare. However, a higher level of mental welfare hasn't been widely considered. There are increasing number of mental health problems in recent years. It becomes the significant factor to restrain the development of companies [2].

Domestic and international studies have already investigated the factors of workers' mental health [2-5]. The literature has suggested that the relevant factors of workers' mental health mainly come from society, profession and workers themselves. First, the common perceptions of mental health care are still outdated [3]. There is widespread prejudice against psychological counseling and treatment. Besides, workers are unwilling to acknowledge their mental health problems, at least until the severity of the symptoms makes it clear to colleagues or family. Consequently, workers fail to seek professional help in early stage and quickly benefit from the early treatment. In addition, several studies have provided that high-intense professional tasks are related to poor mental health outcomes [4]. Meanwhile, $\mathrm{Xu}$ et al. have demonstrated that the mental health condition is positively correlated with negative personal resources [5]. Moreover, coupled with the lack of necessary social support and the ways to decompress, these workers can easily suffer from mental health problems when they engage in high-intensity, monotonous tasks [2]. Based on past studies, personal resources and professional tasks have contributed to new challenges for mental health management. The influence of single factor on workers' mental health has been mainly discussed. However, there are few studies exploring the interrelationship of these factors, especially for manufacturing front-line workers.

Therefore, this study further explores the interrelationship of personal resources, professional tasks and mental health among manufacturing front-line workers using the revised Occupational Stress Inventory [6] (OSI-R) and the Symptom Checklist [7] (SCL-90). By understanding the relationship, companies may be able to devise interventions or strategies to mitigate the negative effects. The study also investigates the applicability of the Conservation of Resource Theory [8] (COR) in the eastern countries like China, which is originally established in the western countries. 


\section{Literature review}

\subsection{Mental health}

Workers' mental health problems have strong negative impact on the companies performance. H.B.English has conceptualized mental health, which is a level of psychological well-being, or an absence of mental health problems[9]. It is the "psychological state of someone who is functioning at a satisfactory level of emotional and behavioral adjustment". From the perspective of positive psychology or holism, mental health may include an individual's ability to enjoy life, and create a balance between life activities and efforts to achieve psychological resilience [9]. In the past few decades, studies have focused on the condition and relevant factors of workers' mental health. Paek et al. found that front-line workers with high psychological capital were more engaged with their work and more likely to display job satisfaction and organizational commitment [10]. Loi et al. thought coworker support reduced foreign workers' mental health problems [11]. Huang et al. implied changes in Chinese migrant workers' mental health by using the SCL-90. And results described that their mental health level was decreasing over time [12]. In addition, Zhong et al. revealed that labor workers' mental health was related to their own psychological adjustment mode and the pressure caused by working environment, working intensity, working conditions and management style. And the SCL-90 was used to measure workers' mental health [13]. These studies have covered the present condition of workers' mental health and its relevant factors, but the functioning mechanism is still lack of study. Therefore, this study further explores the condition of mental health on manufacturing front-line workers using the SCL-90, which has already been wildly used in China.

\subsection{Professional tasks and mental health}

Professional tasks are a set of the working conditions, including role overload, role insufficiency, role ambiguity, role boundary, responsibility, work environment and so on. They have significant impact on work stress, and can be called stressors [14]. A large number of studies have confirmed that professional tasks of workers vary among different companies. Zhang et al. found that professional tasks had significant impact on mental health of the electronic manufacturing front-line workers by using the OSI-R to measure professional tasks [14]. Sharma et al. revealed that work-family conflict had a direct negative relationship with the mental health of female nurses, signifying that the lifestyle of female nurses was that their work and family roles interfered with each other [15]. Ganster et al. stated that there was consistent evidence supporting a link between work stressors and primary Allostatic Load indicators for selfreported affective outcomes such as anxiety, job attitudes, and job-related tension [4]. Chen et al. studied the condition of the work stress among Chinese offshore oil workers using the OSI-R, and the result showed professional tasks were the key factor impacting mental health [16]. In addition, He et al. revealed that manufacturing front-line workers often suffered from the mechanical work with high speed and repetition for a long time [3]. Therefore, in this study, the professional tasks are considered as the major factor of workers' mental health, and the Occupational Research Questionnaire which is the sub-scale of the OSI-R is used to measure workers' mental health because of its good reliability and validity.

\subsection{Personal resources and mental health}

Personal resources refer to the ability of workers to cope with professional tasks. Hobfoll's Conservation of Resource Theory (COR) bridges the gap between environmental and cognitive aspects by introducing behavioral perspectives to analyze the situation [8]. According to the COR model, people suffer from mental health problems due to: (1) the threat of the loss of resources, (2) the actual loss of resources, or (3) a lack of resources gain following the investment of resources[17]. People strive to either acquire or maintain resources that have value for them. The COR model describes how the professional tasks cause mental health problems evidenced by depression, phobic and anxiety due to the loss of personal resources in the process, and it's suitable for this study to use the COR model to explore the interrelationship of personal resources, professional tasks and mental health. The study of college students has appeared that social support mediated the relationship between emotional intelligence and mental health [18]. In the study focusing on medical workers' health, different kinds of coping resources were considered to have different impacts on mental health, and the coping resources were measured by the OSI-R[19]. Above all, studies have suggested that personal resources change the way that external environment impacts mental health. Examination of the relationship between personal resources and mental health may shed light on how individuals respond in certain situations and provide us with some information on how to intervene. Therefore, the personal resources are taken as another significant factor of workers' mental health in this study, and the Personal Resources Questionnaire which is the sub-scale of the OSI-R is also used to measure workers' personal resources because of its comprehensive content.

\section{Materials and methods}

\subsection{Participants}


The front-line workers were investigated from a typical electrical manufacturing company in Zhengzhou by random sampling, which was a leading company of global electrical manufacturing industry with 200,000 workers. The investigation was conducted through asking front-line workers to answer the questionnaires distributed to them. Prior to the data collection informed consent was obtained from the workers.

From March 2016 to May 2016, a total of 480 questionnaires were delivered to front-line workers, and 392 qualified questionnaires remained for analysis during the data cleaning process in this study. Males comprised $54.85 \%$ of the total sample. The majority of participants were the only child ( $82.91 \%$ of the sample). $68.11 \%$ of participants ranged in age from 20 to 29 . And $62.76 \%$ were married at the time the study took place. Only $16.33 \%$ of participants were college graduates. Additionally, workers with countryside origin comprised $73.47 \%$ of the total sample. In summary, The structure of sample was similar to that of company manpower.

\subsection{Measures}

Data was collected by using the OSI-R and the SCL-90 which were proved valid and reliable in past studies. These items were all rated on a 5-point Likert scale. The OSI-R included 140 items, and was divided into 3 sub-scales: Occupational Research Questionnaire (ORQ), Personal Resources Questionnaire (PRQ) and Personal Strain Questionnaire (PSQ) [6]. The ORQ and PRQ were selected to measure workers' professional tasks and personal resources respectively. Besides, mental health was measured by using SCL-90 with possible answers ranging from $1=$ All of the time to $5=$ None of the time [7]. After pretest, Cronbach alphas for three scales were $0.87,0.90$ and 0.88 . Results showed that these scales were suitable for manufacturing front-line workers. In addition, questionnaire also included a brief report form that collected information on demographic characteristics.

\section{Results}

T-test and Pearson correlation analysis were conducted in this study using SPSS 17.0.

\subsection{Description of the variables}

A simple linear transformation was proposed to standardize the collection data of the OPQ and PRQ.The conversion formula was presented below. $x$ stands for the actual score of item; $n$ stands for the total number of items, and $S$ is the standard score [14].

$$
S=\frac{\sum x}{n \times 5} \times 10
$$

After standardized treatment, the descriptive statistics of the variables were shown in table 1 .

Table 1 showed that the average scores of all indicators was significantly different from the national norm except interpersonal sensitivity. In professional tasks, the scores of role overload, role insufficiency and role boundary were higher than the national norm. However, the scores of role ambiguity, responsibility and work environment were below the national norm. All scores of indicators in personal resources were below the national norm. The GSI (M=1.62, $\mathrm{SD}=0.55$ ) of SCL-90 was significantly different from the national norm, and the scores of indicators were much higher than the national norm except interpersonal sensitivity. The results indicated that among manufacturing front-line workers, the condition of mental health and professional tasks were lower than the national average level, and the personal resources were relatively deficient as well.

\subsection{Correlation analysis}

The results of Pearson correlation analysis were shown in table 2, indicating that professional tasks and personal resources were both correlated strongly with mental health. It stated a positive relationship between the indicators of mental health problems and professional tasks (except responsibility), while personal resources and mental health problems were strongly negative correlation. Besides, the correlation coefficients of role overload, social support, role insufficiency and role boundary were obviously higher than other indicators.

Table 1. Descriptive statistics of the variables.

\begin{tabular}{cccccc}
\hline & Factor & Mean & SD & Norm & t \\
\hline \multirow{5}{*}{ professional tasks } & Role overload & 6.42 & 1.06 & $5.79 \pm 1.16$ & $11.75^{* * *}$ \\
& Role insufficiency & 6.59 & 0.88 & $6.05 \pm 1.38$ & $12.28^{* * *}$ \\
& Role ambiguity & 5.42 & 1.17 & $5.64 \pm 2.11$ & $-3.79^{* * *}$ \\
& Role boundary & 5.29 & 1.12 & $4.96 \pm 1.02$ & $5.83^{* * *}$ \\
personal resources & Responsibility & 4.82 & 1.29 & $4.93 \pm 1.27$ & $-1.64^{*}$ \\
& Work environment & 4.58 & 1.74 & $5.18 \pm 1.47$ & $-6.85^{* * *}$ \\
& Recreation & 4.85 & 1.21 & $5.48 \pm 1.09$ & $-10.45^{* * *}$
\end{tabular}




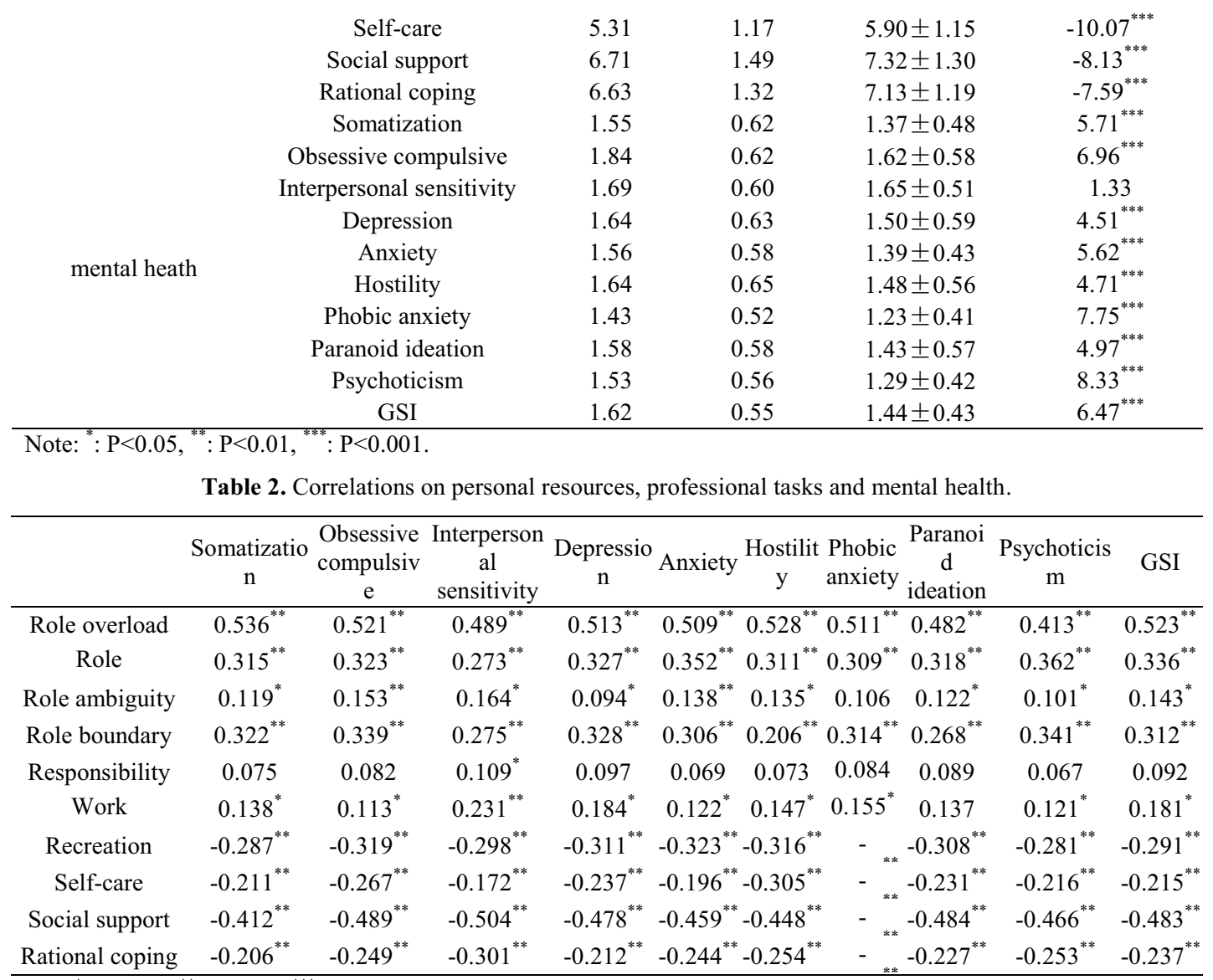

Note: ${ }^{*}: \mathrm{P}<0.05,{ }^{* *}: \mathrm{P}<0.01,{ }^{* * *}: \mathrm{P}<0.001$.

\section{Discussion}

To study the mental health condition of the manufacturing front-line workers, the interrelationship of personal resources, professional tasks and mental health was explored in this study.

The questionnaire about professional tasks of 392 front-line workers indicated that the average scores of role overload, role insufficiency and role boundary were higher than the national norm. On the contrary, the scores of role ambiguity, responsibility and work environment were lower than the national norm. Results implied that manufacturing front-line workers were lack of responsibility for heavy tasks. However, the duty of job was defined clearly and work environment was tolerable. Essentially, many manufacturing companies were labor intensive companies in China. The consecutive work for front-line workers exceeded 10 hours a day. Companies usually implemented shift work operation and asked workers to work overtime on weekends or holidays. The manufacturing front-line workers engaged in high-intensity, monotonous tasks day by day, which led to an increase in the number of mental health problems in recent years.

In this study, the scores of all indicators in personal resources were below the national norm. It implied that workers lacked the necessary social support and ways to relieve stress when they engaged in the high-intensity tasks. The indicators included in this study depicted the individual conditions of the COR model. The high-intensity tasks caused an actual loss of resources due to the multiple demands of work, and the fear of resource loss created mental health problems. The theory of conservation of resources advocated that personal resources were exploited in the process of relieving stress. Thus, it was imperative for managers to take workers' mental welfare into frame policies to promote workers' physical and mental health, so as to achieve high quality job.

The GSI of SCL-90 in this study was significantly different from the national norm, and the score of its indicators were much higher than the national norm except interpersonal sensitivity. It implied that the condition of mental health among manufacturing front-line workers was poor. And it's below the national average level. Combined with the present survival status of manufacturing workers, high-intensity tasks and harsh management style can be considered as the major factors of the mental health problems. Because manufacturing front-line workers with heavy tasks had fewer opportunities for human interaction. Moreover, in order to maximize profits, companies regarded cheaper labor as a 
tremendous competitive advantage. Thus, they usually formulated draconian management system and treated workers as machines. Under the special working condition and inflexible management system, workers endured excessive work stress and lacked the way to release their emotion. These factors will inevitably led to mental imbalance.

Correlation analysis indicated a negative relationship between the indicators of mental health and professional tasks (except responsibility), while personal resources and mental health were significantly positive correlation. It implied that the mental health problems were severer in population with heavier tasks and less personal resources. In professional tasks, the correlation coefficients of role overload, role insufficiency and role boundary were obviously higher than other factors. In addition, social support was the key indicator of the correlation between personal resources and mental health. Because manufacturing front-line workers were usually away from home and lacked of proper care from relatives and friends. Meanwhile, their experience of solving severe mental health problems was insufficient. So that, the support from organization, colleagues and friends had a considerable effect on workers' mental health. According to the results, managers can improve workers' mental health by increasing mental welfare. For instance, increasing entertainment facilities in the factory, organizing mental health-care activities, implementing job-rotation regularly and improving the psychological help system. Moreover, recruiting local workers could also improve their personal resources, which was the major factor of workers' mental health.

\section{Conclusion}

The study has explored interrelationship of personal resources, professional tasks and mental health through the analysis of the questionnaire. Several tentative conclusions may be drawn: (1) The mental health problems are severer among manufacturing front-line workers with heavier tasks and less personal resources. (2) The indicators of personal resources are below the national norm. In professional tasks, the scores of role overload, role insufficiency and role boundary are higher than the national norm. However, the scores of role ambiguity, responsibility and work environment are lower than the national norm. The average scores of SCL-90 are much higher than the national norm except interpersonal sensitivity. (3) Personal resources and mental health are significantly positive correlation, while the indicators of professional tasks (except responsibility) have a significantly negative impact on mental health. So, it is an important way to ensure the front-line workers' mental health by improving workers' mental welfare, like enhancing personal resources and reducing professional tasks.

\section{References}

1. NBS. China Statistical Yearbook (Chin Stats Press, Beijing, 2014)

2. Z.G. Lin, F.M. Fan, J. Wu, Chin. J. Health. Psy, 20, 1345 (2012)

3. J.L. He, R. Zhao, Talent Exploitation, 7, 19 (2010)

4. D.C. Ganster, C.C. Rosen, J. Mgmt, 39, 1085 (2013)

5. M.J. Xu, X.N. Huang, Z.Y. Feng, J. Environ \& Occup. Med, 33, 134 (2016)

6. S.H. Osipow, PAR-Psy. Assess. Res. Inc, (1998)

7. L.R. Derogatis, R.S. Lipman, K. Rickels, Behav. Sci, 19, 1 (1974)

8. S.E. Hobfoll, Appl. Psy, 50, 337 (2001)

9. H.B. English, A.C. English, 115, 957 (1958)

10. S. Paek, M. Schuckert, T.T. Kim, Int. J. Hosp. Mgmt, 50, 9 (2015)

11. R. Loi, O.K.Y. Ao, A.J. Xu, Int. J. Hosp. Mgmt, 36, 23 (2014)

12. S.L. Huang, W.J. Hou, M. Zhang, Acta Psy. Sinica, 47, 466 (2015)

13. Q.Y. Zhong, K. Li, G.Q. Li, Chin. J. Health. Psy, 12, 1815 (2013)

14. Z.W. Zhang, B. Liu, B.F. Li, Ind. Eng. Mgmt, 18, 141 (2013)

15. J. Sharma, R.L. Dhar, A. Tyagi, Appl. Nurs. Res, 30, 268 (2016)

16. W. Chen, T. Wong, T. Yu, Work. Stress, 17, 287 (2003)

17. L.M. Little, D.L. Nelson, M.J. Quade, J. Vocat. Behav, 79, 724 (2011)

18. M. Zeidner, G. Matthews, Pers. Indiv. Diff, 99, 196 (2016)

19. W. Zhu, Q.S. Jiang, Z.M. Wang, J. Zhengzhou. Univ. (Med. Sci), 43, 468 (2008) 